\title{
Theoretical Bounds on Electron Energy Filtering in Disordered Nanomaterials
}

\author{
Amro Dodin ${ }^{\dagger}$ Brian Aull, ${ }^{\ddagger}$ Roderick R. Kunz, ${ }^{\ddagger}$ and Adam P. Willard ${ }^{*} \dagger$ \\ $\dagger$ Department of Chemistry, Massachusetts Institute of Technology, Cambridge, \\ Massachusetts 02139, United States \\ $\ddagger$ Lincoln Laboratory, Massachusetts Institute of Technology, Lexington, Massachusetts \\ 02421, United States \\ E-mail: awillard@mit.edu
}

\begin{abstract}
Nanostructured materials can act as electron energy filters by funneling thermally broadened electrons through discrete energy levels. To date, all implementations of this effect have used a single nanostructure as an electron filter, thereby avoiding the potential influence of heterogeneity that would be present in any device scale application. In this study, we develop a theoretical model of the electron filtering properties of nanostructured materials that explicitly includes the effects of thermal broadening and size polydispersity on the heterogeneity of nanostructure energy levels. We find that under certain conditions quantum dot solids can perform as effective electronic energy filters, but that materials comprised of quantum wires or quantum wells have continous transverse electronic bands that make them ineffective for this purpose. We identify a material specific length scale parameters, $L_{\text {crit }}$, that specifies the maximum mean quantum dot size that can yield effective energy filtering. Moreover, we show that energy filtering materials comprised of QDs with size near $L_{\text {crit }}$ are maximally robust to heterogeneity in quantum dot size, tolerating variations $\sim 10 \%$ of the mean size. The
\end{abstract}


length scale $L_{\text {crit }}$ can be estimated directly from the widely-tabulated density of states effective mass and show that semiconductors with light conduction band electrons, such as III-V type materials InSb and GaAs are the most forgiving for energy filtering applications. Taken together, these results provide a practical set of quantitative design principles for semiconductor electron filters.

\section{Introduction}

Electronic currents are used to generate and carry signals in a wide range of technological applications. In some cases, this signal can be obscured by energetic uncertainty in the electronic population. One way to reduce this uncertainty is to pass the flow of electrons through an energy filter. In this manuscript we study the electron filtering properties of nanoparticle composite materials, which have been proposed as potentially effective energy filters. ${ }^{117]}$ We highlight that although transport through the discrete energy levels of individual nanoparticles reduces energetic uncertainty, variations between nanoparticles in the material provides an additional source of energetic broadening. We present a theoretical model that quantifies the influence of these effects in materials made up of quantum dots, quantum wires, or quantum wells. We then apply this model to identify a set of nanomaterial design parameters for effective electron filters.

In most materials, uncontrolled thermal broadening is the dominant source of uncertainty in electron energies. The unwanted effects of this uncertainty can therefore be reduced or eliminated by lowering the temperature of the electron source. For example, in simple metallic electrodes the thermal broadening around the Fermi energy can be reduced from about $60 \mathrm{meV}$ at room temperature to about $15 \mathrm{meV}$ at $T=77 \mathrm{~K}$. While this approach to reducing electronic uncertainty is straightforward, it also imposes constraints on operating conditions that are expensive and impractical. If electron filtering materials could achieve a similar level of energetic uncertainty reduction under ambient conditions, then they would dramatically expand the scope of these applications and catalyze further technological and 
scientific advances.

Here, we focus our motivation on a specific probe of chemical structure known as inelastic electron tunneling spectroscopy (IETS). ${ }^{8}[10$ In this technique, vibrational spectra are measured by transmitting a flow of electrons through a sample that is contained within a tunneling junction. Tunneling electrons are scattered when their energies are resonant with molecular vibrational frequencies, and in this way vibrational spectra are encoded in the voltage dependence of the tunneling current.11-17 Unfortunately, achieving the spectral resolution required to reliably distinguish molecular vibrational signatures $(\sim 0.5 \mathrm{meV})^{18-20}$ presents a technical challenge that has limited current experiments to cryogenic conditions. Nonetheless, by constructing custom tunneling junctions that contain specific analytes, IETS has been used to measure the vibrational spectra of organic and inorganic molecules, 2127 to chemically analyze materials adsorbed on surfaces in scanning tunneling microscopy experiments,,$\frac{2829}{21}$ and even to sequence DNA. .30131

The ability to extend IETS to ambient conditions would enable the development of a new generation of compact and efficient solid-state chemical sensors. Specifically, it has been suggested that with IETS, simple open tunneling junctions can be transformed into general optical-free chemical sensors. ${ }^{32134}$ Moreover, the use of low cost Si CMOS compatible materials could enable precise design of these sensors so that they can be made to match the specificity of longwave infrared vibrational spectroscopy and be incorporated as nanoscale elements of solid state electronic circuits, for use in applications such as continuous-flow chemical reactors, microfluidic channels and chemical olfaction devices. Incorporating energy filtering materials into the design of open tunneling junctions is essential for expanding the temperature range for IETS and enabling these applications.

Nanostructured materials accomplish energy filtering by restricting electrons to discrete quantized energy levels, rather than within a continuum. However, all current current demonstrations of electron filtering in these materials have utilized single nanostructures. ${ }^{[16135}$ While these demonstrations provide a useful proof-of-concept, they fall short of a practical solution 
to the energy filtering problem because devices based on single nanostructures are difficult to construct and yield very weak signals. More practical (device scale) energy filtering nanomaterials will need to incorporate the combined effect of many individual nanostructures.

The electronic energy levels of nanomaterial made up of many individual nanostructures will include broadening due to variations in nanostructure size. This is illustrated schematically in Fig. 1, which depicts the electronic energy landscape of a hypothetical onedimensional array of quantum wells made of the same material but with varying widths. This type of population heterogeneity manifests as inhomogeneous broadening in the absorption spectra of nanomaterials, such as quantum dot (QD) solids. $\stackrel{36}{-38}$ Variations in the nanostructure population typically originate from a lack of precision in the synthesis of nanostructures and can thus be controlled, to some extent, by tuning synthetic conditions.

The dependence of energy level positioning on nanostructure size is a consequence of quantum confinement and has the general effect that smaller structures have higher energy ground states and more widely spaced energy levels, as depicted for the quantum wells in Fig. 1. This effect contributes to two sources of size dependent energy level broadening: (1) homogeneous broadening due to spontaneous thermal excition into low-lying excited states, and (2) inhomogeneous broadening due to variations in the ground state energy levels of nanostructures with different sizes. Minimizing energy level broadening of a nanomaterial requires balancing a competition between these two sources, which exhibit opposing dependencies on nanostructure size. Specifically, as nanostructures become smaller the homogeneous broadening decreases, due to the widening gap between ground and excited state energies (which exponentially supresses thermal excitation). At the same time, the inhomogeneous broadening tends to increase due to a larger relative sensitivity of energy level positions to size variations.

In the section below, we describe the theoretical methodology used in this work. First, we discuss the calculation of energy levels in quantum dots and quantum wells, and we outline a Monte-Carlo method for sampling energy levels. Then, in the Results section, we discuss 


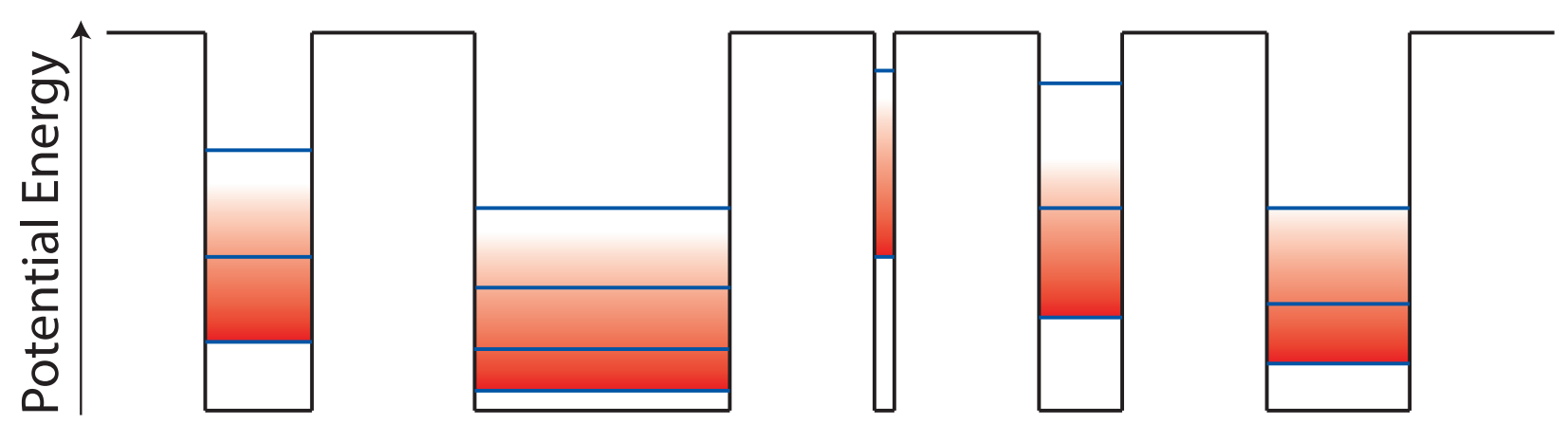

Figure 1: Schematic illustration of the electronic energy landscape of a one-dimensional array of quantum wells with different widths. For each well, the few lowest localized eigenstate energies are shown as blue horizontal lines. Differently sized wells have differing state energies, leading to inhomogeneous broadening. The relative probability for thermal excitation is represented by shading, with darker red corresponding to larger probability. Thermal excitation within each well leads to the population of higher lying states, producing homogeneous thermal broadening.

the predicted specifications for nanostructure electron filters and derive analytical design principles for suppressing thermal broadening. Finally, we conclude by outlining the current outlook and required developments for realistic room temperature nanostructed electron filters.

\section{Theoretical Methods}

We model a nanomaterial as a composite of independent homogeneously broadened nanoparticles with a distribution of different sizes. Individual nanoparticles are treated as idealized systems and describe chemical composition using the effective mass approximation. Although this model is simple, it effectively captures the competition between homogeneous and inhomogeneous broadening and thus provides a theoretical basis for generating design principles for these materials. In this section, we first describe our simple theoretical approach for describing the energy levels of individual nanoparticles and then we describe our method for sampling homogeneous and inhomogeneous broadening. 


\section{Modeling the Energy Levels of Individual Nanostructures}

We model nanostructures using the effective mass envelope approximation, which has been widely used for estimating the electronic energy levels of nanostructures. ${ }^{39}$ In this approximation, the effect of atomic structure on the electronic wavefunction is neglected. Instead, electrons are treated as free particles, with a material-specific effective mass, that are constrained by a potential energy function that defines the shape and size of the nanostructure. This efficient approach to computing confinement effects on electronic energy levels cann be applied to a broad range of materials and nanostructure morphologies, thereby distilling the role of material specific properties into a single, widely tabulated effective mass parameter. 40141

Our choice of modeling approach is motivated by computational efficiency. More sophisticated methods, such as those based on first principles electronic structure calculation, are more accurate but much more computationally expensive. Even with access to large computational resources these methods are limited to nanoparticles with a small number of atoms (nanoparticle diameter $\sim 1 \mathrm{~nm}$ ), and must be performed separately for every nanostructure size, shape, and chemical composition. $[245$ These methods are prohibitively expensive for the scope of this study, which requires energy level calculations for $\sim 10^{5}$ different nanoparticles.

\section{Quantum Dots}

We model the electronic energy landscape of a quantum dot as a standard particle in a cubic box with sidelength $L$. The energy levels for electrons in this potential are given by,

$$
E(\boldsymbol{k})=\frac{\hbar^{2}}{2 m^{*}} k^{2}
$$

where $m^{*}$ is the effective mass of the electron, $\hbar$ is the reduced Planck constant and $\boldsymbol{k}=$ $\frac{\pi}{L}\left(n_{x} \hat{x}+n_{y} \hat{y}+n_{z} \hat{z}\right)$ is the quantized wave vector with positive integer quantum numbers $n_{i}$, and $k=\frac{\pi}{L} \sqrt{n_{x}^{2}+n_{y}^{2}+n_{z}^{2}}$ is the wavector magnitude. 


\section{Quantm Wells and Quantum Wires}

A quantum well is a nanostructure that is only confined in one dimension. We model the confining potential of a quantum well as two parallel infinite barriers, separated by distance $L$, and quasi-infinite (i.e., periodically replicated) in the two transverse dimensions. The electronic Hamiltonian for such a system can be separated into a periodic transverse

component $H(x, y)$ and a one dimensional confinement Hamiltonian $H(z)$, enabling the separate treatment of the discrete and periodic energy spectra.

We model the confined dimension as a particle with effective mass $m^{*}$ in a one-dimensional box of length $L$. The energy levels for this model are given by,

$$
E_{\|}(n)=\frac{\hbar^{2} \pi^{2}}{2 m^{*} L^{2}} n^{2}
$$

where $n=1,2, \ldots$

In general, modeling the energy levels of the periodic dimensions requires material specific information, such as the chemical identity of the constituent atoms and the underlying lattice structure. However, in most materials at room temperature, thermal excitation tends to drive electrons into states that are near in energy to the conduction band edge. Based on this tendency, we formulate the electronic dispersion relation using the standard parabolic bands approximation (i.e., as a second order Taylor expansion in $\boldsymbol{k}$ ). The resulting expression for the transverse energy levels is given by,

$$
E_{\perp}\left(\boldsymbol{k}_{\boldsymbol{x} \boldsymbol{y}}\right)=\frac{\hbar^{2}}{2 m^{*}} k_{x y}^{2}
$$

where $\boldsymbol{k}_{\boldsymbol{x} \boldsymbol{y}}$ is the two-dimensional wave-vector in the transverse $x-y$ plane. The resulting energetic density of states is equivalent to a free particle with effective mass $m^{*}$ and is effective for a wide range of materials.

A quantum wire is like a quantum well, but confined in two dimensions instead of one. 
We model the energy levels of a quantum wire as a particle in a square box that is quasiinfinite in the one transverse direction. By separating the confined and periodic components, a quantum wire can therefore be formulated analogously to that of our model quantum well described above.

The continuous free particle dispersion in the transverse dimensions of quantum wells and quantum wires is problematic for energy filtering applications. In fact, this continuous manifold of energy states are equivalent to the energetic structure that leads to thermal broadening in the bulk material. To illustrate this, we consider a quantum well and note that a quasi-free particle with a quadratic energy dispersion as described by Eq. (3) leads to a Gaussian thermal distribution of electrons

$$
P\left(\boldsymbol{k}_{\boldsymbol{x} \boldsymbol{y}}\right) \propto e^{-\frac{\hbar^{2} k_{x y}^{2}}{2 m^{*} k_{B} T}}
$$

where $k_{B}$ is the Boltzmann constant and $T$ is the temperature. This is precisely the thermal broadening that an electron filter seeks to attenuate. As a result, pristine quantum wires and quantum wells with no defect or trap states cannot act as effective electron filters and the remainder of this study will be dedicated to 0D quantum dot systems. We note, however, that an experimental study on quantum well devices have shown resonant electron filtering through discrete energy levels similar to quantum dots. ${ }^{[6}$ This puzzling observation suggests the involvement of some quantum dot-like localized defect state or a device-specific transverse momentum selection mechanism that is beyond the scope of our study.

\section{Statistical Sampling}

We model the effects of homogeneous and inhomogeneous broadening by sampling an equilibrium ensemble of electronic energy levels drawn from a distribution of QD sizes. To sample

this ensemble, we use a Metropolis-Hastings Monte Carlo (MHMC) sampling scheme. ${ }^{46}$ This stochastic algorithm is widely used to study the equilibrium properties of thermodynamic 
systems as it can efficiently sample from a distribution using only the relative probability of pairs of states. The specific Metropolis-Hastings Algorithm used in this study is summarized in Scheme 1 and an example of the sampling is shown in Fig. 2. Here, we have assumed a Gaussian size distribution with mean $\bar{L}$ and standard deviation $\sigma_{L}$. To efficiently sample the electronic energy levels of a quantum dot with size $L$, we define a cut-off state $n_{\text {cut }}$ above which thermal population can be neglected. In this study, $n_{\text {cut }}$ is defined as the state where the probability of thermal excitation from the ground state is $\frac{P\left(\left(n_{\text {cut }}, 1,1\right)\right)}{P((1,1,1))}=\exp \left(\frac{-E\left(\left(n_{\text {cut }}, 1,1\right) ; L\right)-E((1,1,1) ; L)}{k_{B} T}\right)<10^{-6}$.

0 Sample initial energy level by choosing a particle size $L$ from a Gaussian distribution $P(L)$, and then choosing a quantum number $\boldsymbol{n}=\left(n_{x}, n_{y}, n_{z}\right)$ from a uniform distribution with $n_{i}<n_{\text {cut }}$.

1. Sample a trial energy level by choosing a trail particle size $L_{\text {trial }}$ from $P(L)$ and a quantum number $\boldsymbol{n}_{\text {trial }}$ from a uniform distribution with $n_{i}<n_{\text {cut }}$.

2. Accept the trial energy level with probability $P_{a c c}=\min \left\{e^{-\frac{E\left(\boldsymbol{n}^{\prime} ; L_{\text {trial }}\right)-E(\boldsymbol{n} ; L)}{k_{B} T}}, 1\right\}$.

- If accepted: Set $L=L_{\text {trial }}$ and $\boldsymbol{n}=\boldsymbol{n}_{\text {trial, }}$, otherwise leave $L$ and $\boldsymbol{n}$ unchanged.

3. Add $E(\boldsymbol{n} ; L)$ to the energy level ensemble. Repeat steps $1-3$ until the ensemble is sufficiently sampled.

Scheme 1: MHMC Algorithm used to sample energies of electrons harvested from discrete states in nanostructure ensemble.

\section{Results and Discussion}

We quantify the energy filtering capabilities of a given nanomaterial by first sampling its equilibrium energy level distribution, following the Monte Carlo procedure described in Scheme 1 and illustrated in Fig. 2. We then compare the width of that distribution to the standard thermal broadening (i.e., $k_{\mathrm{B}} T$ ) of the analogous bulk material. We define the width of the sampled energy level distribution in terms of its variance, $\lambda=\left\langle E^{2}\right\rangle-\langle E\rangle$, where the angle brackets represent the average over an equilibrium ensemble. We define a model 
A.
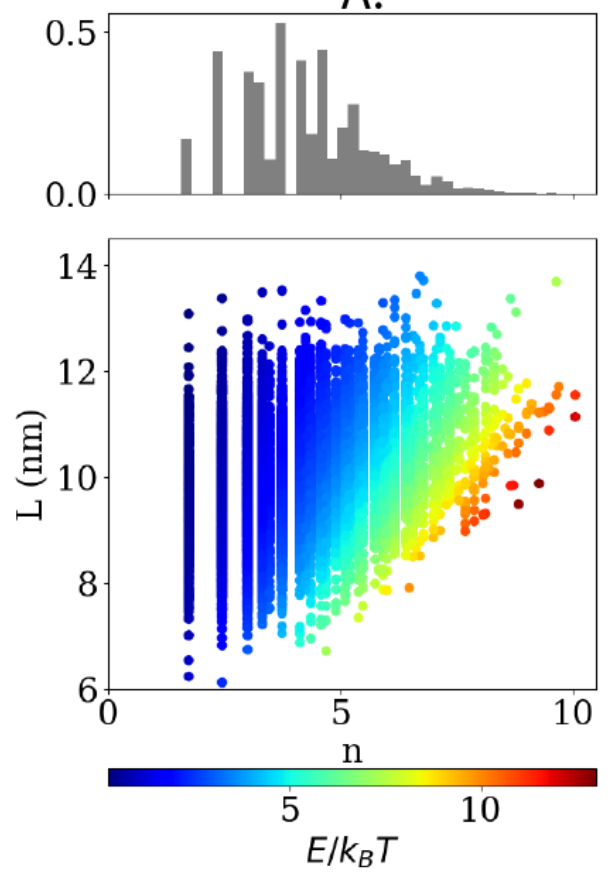

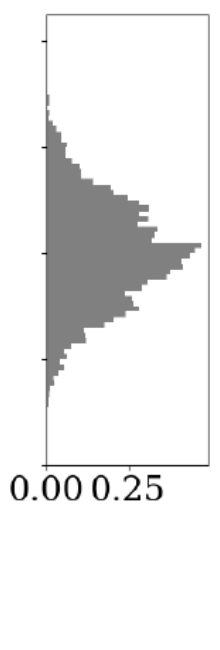

B.

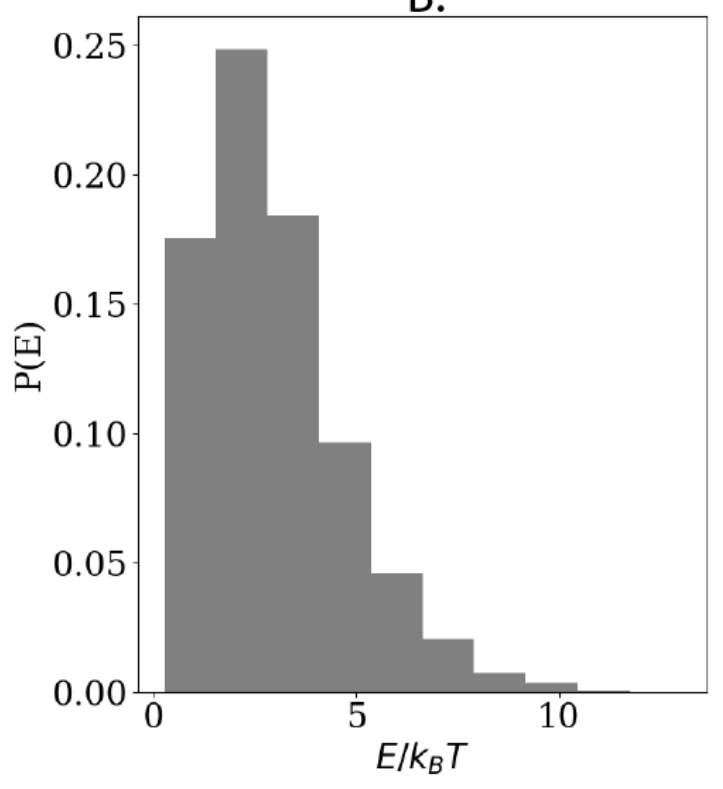

Figure 2: Example output of the energy level sampling described in Scheme 1. Results are shown for $10^{5}$ sampled energy levels in a nanomaterial of Si QDs with $\bar{L}=10 \mathrm{~nm}, \sigma=1 \mathrm{~nm}$, and $T=275 \mathrm{~K}$. (A) A scatter plot of the sampled values of $L$ and $n=\sqrt{n_{x}^{2}+n_{y}^{2}+n_{z}^{2}}$ with the projected probability distributions shown as histograms on the periphery of the scatter plot. Scatter plot points are colored to reflect the energy level for that combination of $L$ and $n$. (B) The probability distribution for energy levels generated from the sampling. 
nanomaterial by specifying the size distribution of the nanoparticles that comprise it. Here, we consider QD solids with a Gaussian distribution of sizes,

$$
P(L) \propto \exp \left[(L-\bar{L})^{2} / 2 \sigma^{2}\right]
$$

where $\bar{L}$ and $\sigma$ are the mean and variance of the QD size distribution, respectively.
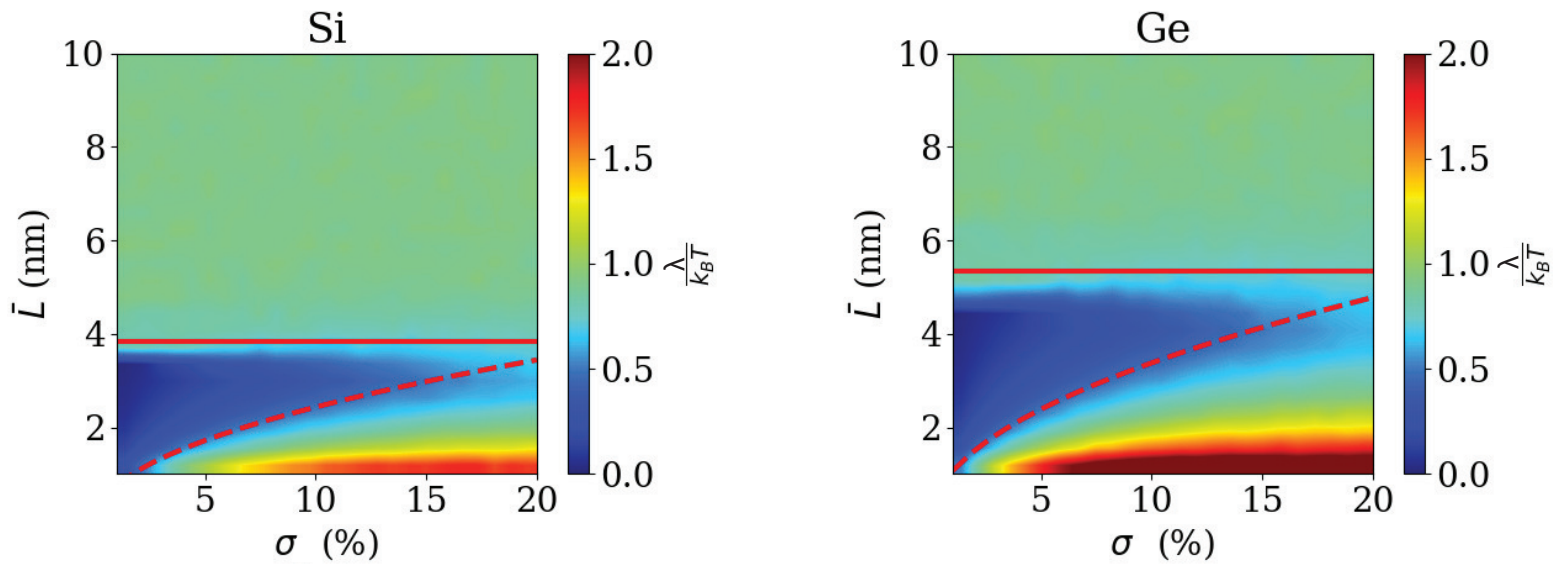

Figure 3: The effective energy filtering properties of QD-based nanomaterials made of $\mathrm{Si}$ and Ge with varying mean $\mathrm{QD}$ size, $\bar{L}$, and relative population heterogeneity, $\sigma$. The width of the electronic energy level distribution relative to that of the bulk thermal distribution, i.e., $\lambda / k_{\mathrm{B}} T$, is indicated by shading. Distributions were sampled using the Monte Carlo algorithm at $T=274 K$. Solid red lines show the critical QD size $L_{\text {crit }}$ from Eq. (7). The dashed lines give the size variability at which heterogeneous broadening $\lambda_{\text {het }}=\frac{1}{2} k_{\mathrm{B}} T$. The region between these two lines roughly corresponds to the nanomaterial parameters that function as effective electron filters.

The general energy filtering properties for these materials can be understood by considering simple Si- and Ge-based QD solids. The energy filtering properties of these materials, as computed from our model, are presented in Fig. 3. We observe that both materials exhibit classical behavior when QDs are above a material specific critical size, which we denote as $L_{\text {crit }}$. We find that $L_{\text {crit }}$ is larger for Ge-based systems $\left(L_{\text {crit }}^{(\mathrm{Ge})} \approx 5.33 \mathrm{~nm}\right)$ than for Si-based systems $\left(L_{\text {crit }}^{(\mathrm{Si})} \approx 3.84 \mathrm{~nm}\right)$. We attribute this difference in $L_{\text {crit }}$ to differences in the effective masses of the materials $\left(m_{\mathrm{Ge}}^{*}=0.56 m_{e}\right.$ and $m_{\mathrm{Si}}^{*}=1.08 m_{e}$, where $m_{e}$ is the mass of an electron in vacuum). Below $L_{\text {crit }}$, these materials can behave as effective electron filters (i.e., 
$\lambda<k_{\mathrm{B}} T$ but only for certain combinations of $\bar{L}$ and $\sigma$.

The qualitative change in the relative width of the electron energy level distribution that we observe at $L_{\text {crit }}$ is a consequence of heterogeneous thermal broadening in individual QDs. This can be seen by considering the energetic spacing between the lowest two electronic energy levels of a QD,

$$
E_{2}-E_{1}=\frac{3 \hbar^{2} \pi^{2}}{2 m^{*} L^{2}} .
$$

If this energy spacing is larger than $k_{\mathrm{B}} T$, then the the majority of the population is confined to the single $E_{1}$ level. Below this spacing, spontaneous thermal excitations lead to additional sources of energetic broadening. We can therefore define a natural lengthscale by setting the left-hand side of Eq. 6 to $k_{\mathrm{B}} T$. By doing so, we arrive at an estimate for the critical QD size,

$$
\bar{L}_{c r i t}=\sqrt{\frac{3 \hbar^{2} \pi^{2}}{2 m^{*} k_{B} T}},
$$

which we indicate with a solid red line in Fig. 3

The formula in Eq. 7 provides a convenient design rule for predicting the minimum required nanostructure size for a arbitrary materials using only the density of states and effective mass. In Fig. 4 we plot the predicted value of $L_{\text {crit }}$ as a function of effective mass and indicate the location of several common semiconducting materials. We find that semiconductors with light conduction band electrons are predicted to be particularly effective electron filters. Possible examples include the III-V type materials with GaAS showing electron filtering up to $\sim 27 \mathrm{~nm}$ and InSb being effective at surprisingly large sizes $\sim 60 \mathrm{~nm}$.

By analyzing the region below $L_{\text {crit }}$ in Fig. 3 we find that certain combinations of $L$ and $\sigma$ can lead to an increase in energy level broadening relative to that of the bulk. This behavior is observed specifically in nanomaterials with larger values of polydispersity. In fact, in both Si- and Ge-based QD materials, we find that inhomogeneous broadening in the ensembles with the smallest means can lead to a spread of electronic energies that are significantly larger than the bulk by a factor of 2. Notably, the relative energetic broadening becomes 


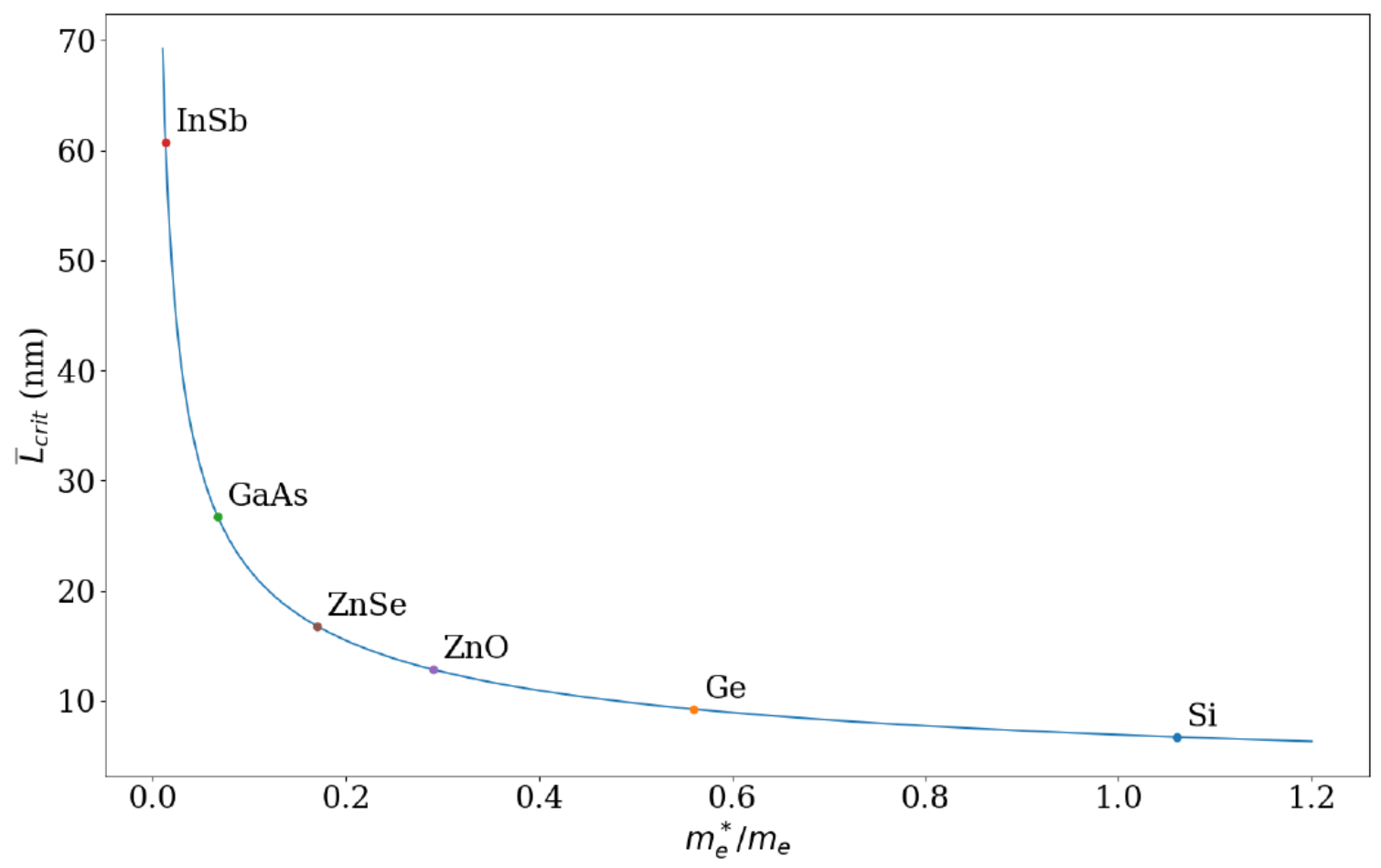

Figure 4: Estimate of the mean QD size for effective electron filtering in nanomaterials of common semiconductor composition, as derived from Eq. (7).

less sensitive to QD size variability for nanostructures with mean QD size closer to that of $\bar{L}_{\text {crit. }}$. This suggests that energy filtering nanomaterials with QD sizes less than but close to $L_{\text {crit }}$ are the most robust to synthetic variations in QD size.

We can understand this increase in energy level broadening by considering the effect of size uncertainty on the standard deviation of the population of lowest energy levels. Specifically, the inhomogeneous broadening of the lowest QD energy level due to size variations can be estimated as,

$$
\lambda_{\text {het }}=\frac{\hbar^{2} \pi^{2}}{2 m^{*} \bar{L}^{2}} \sigma,
$$

which we indicate with a dashed red line in Fig. 3. We find that this relationship effectively delineates regions of increased and decreased energy level broadening for both Si- and Gebased QD nanomaterials. Remarkably, Eqs. 7 and 8 serve to bound the region of effective electron filtering materials. 


\section{Conclusion}

Using Monte-Carlo simulations and analytical estimates we have obtained a set of quantitative design principles for QD-based electron filtering materials. Each class of nanomaterials is shown to have a critical average QD size, below which thermal excitation is effectively suppressed by the discrete energy levels in the QDs. We find that semiconductors with light conduction band electrons, such as the Group III-V materials InSb and GaAs, maximize this critical size and are therefore the most forgiving materials for filter fabrication. Moreover, the analytical results presented in this study allow for the straightforward prediction of the materials and nanostructure sizes effective at reducing thermal broadening. Furthermore, we are able to bound the allowable size heterogeneity in the ensemble for a desired level of performance.

Encouragingly, some of these nanostructures can be reliably fabricated with existing technologies. For example, molecular beam epitaxy techniques are widely used to fabricate

nanostructures on the order of single nanometers with monolayer precision $\underline{47 / 48}$ and are able to integrate them into larger devices. In addition, some materials have sufficiently large critical length scales and are sufficiently robust to size heterogeneity to enable colloidal synthesis techniques that can produce quantum dots $\sim 1-10 \mathrm{~nm}$ with size variability $\sim 10-20 \%$. $\frac{49}{53}$ This indicates that designing nanostructured electron filters may be possible with existing fabrication methods.

\section{Acknowledgements}

This work was funded through Lincoln Labs by Air Force Contract FA8702-15-D-0001. A.D. was support in part by the Natural Science Scholarship Doctoral program (NSERC PGS D). 


\section{References}

(1) van der Vaart, N. C.; Godijn, S. F.; Nazarov, Y. V.; Harmans, C. J. P. M.; Mooij, J. E.; Molenkamp, L. W.; Foxon, C. T. Resonant Tunneling Through Two Discrete Energy States. Phys. Rev. Lett. 1995, 74, 4702-4705.

(2) Tung, H.-H.; Lee, C. P. A novel energy filter using semiconductor superlattices and its application to tunneling time calculations. IEEE Journal of Quantum Electronics 1996, 32, 2122-2127.

(3) Zhuang, L.; Guo, L.; Chou, S. Y. Silicon single-electron quantum-dot transistor switch operating at room temperature. Appl. Phys. Lett. 1998, 72, 1205-1207.

(4) Postma, H. W. C.; Teepen, T.; Yao, Z.; Grifoni, M.; Dekker, C. Carbon Nanotube Single-Electron Transistors at Room Temperature. Science 2001, 293, 76-79.

(5) Bahk, J.-H.; Bian, Z.; Shakouri, A. Electron energy filtering by a nonplanar potential to enhance the thermoelectric power factor in bulk materials. Phys. Rev. B 2013, 87, 075204.

(6) Bhadrachalam, P.; Subramanian, R.; Ray, V.; Ma, L.-C.; Wang, W.; Kim, J.; Cho, K.; Koh, S. J. Energy-filtered cold electron transport at room temperature. Nature Communications 2014, 5, 4745.

(7) Beckers, A.; Thewissen, M.; Sorée, B. Energy filtering in silicon nanowires and nanosheets using a geometric superlattice and its use for steep-slope transistors. Journal of Applied Physics 2018, 124, 144304.

(8) Jaklevic, R. C.; Lambe, J. Molecular Vibration Spectra by Electron Tunneling. Phys. Rev. Lett. 1966, 17, 1139-1140.

(9) Salace, G.; Patat, J. M. Tunneling spectroscopy possibilities in metal-oxide- 
semiconductor devices with a very thin oxide barrier. Thin Solid Films 1992, 207, 213-219.

(10) Lye, W.-K.; Hasegawa, E.; Ma, T.-P.; Barker, R. C.; Hu, Y.; Kuehne, J.; Frystak, D. Quantitative inelastic tunneling spectroscopy in the silicon metal-oxide-semiconductor system. Appl. Phys. Lett. 1997, 71, 2523-2525.

(11) Baratoff, A.; Persson, B. N. J. Theory of the local tunneling spectrum of a vibrating adsorbate. Journal of Vacuum Science \& Technology A 1988, 6, 331-335.

(12) Galperin, M.; Ratner, M. A.; Nitzan, A. Molecular transport junctions: vibrational effects. J. Phys.: Condens. Matter 2007, 19, 103201.

(13) Walczak, K. Vibrational features in inelastic electron tunneling spectra. Chemical Physics 2007, 333, 63-68.

(14) Jiang, J.; Kula, M.; Luo, Y. Molecular modeling of inelastic electron transport in molecular junctions. J. Phys.: Condens. Matter 2008, 20, 374110.

(15) Horsfield, A. P.; Tong, L.; Soh, Y.-A.; Warburton, P. A. How to use a nanowire to measure vibrational frequencies: Device simulator results. Journal of Applied Physics 2010, $108,014511$.

(16) Liang, Y. Y.; Chen, H.; Mizuseki, H.; Kawazoe, Y. Gate-controlled current and inelastic electron tunneling spectrum of benzene: A self-consistent study. J. Chem. Phys. 2011, $134,144113$.

(17) Dash, L. K.; Ness, H.; Verstraete, M. J.; Godby, R. W. Functionality in single-molecule devices: Model calculations and applications of the inelastic electron tunneling signal in molecular junctions. J. Chem. Phys. 2012, 136, 064708. 
(18) Kudo, K.; Okazaki, C.; Kuniyoshi, S.; Tanaka, K. Inelastic Electron Tunneling Spectroscopy of Langmuir-Blodgett Monolayers on Silicon Substrate. Jpn. J. Appl. Phys. 1991, 30, 1452 .

(19) Bommisetty, V.; Bhandari, S.; Karmacharya, R. L.; Rislov, D. A.; Mileham, R. D.; Galipeau, D.; Mileham, R. D.; Galipeau, D. W. Gas Sensing Based on Inelastic Electron Tunneling Spectroscopy. IEEE Sensors Journal 2008, 8, 983-988.

(20) Zadeh, Y. H.; Durrani, Z. A. K. Inelastic electron tunneling spectroscopy for molecular detection. Journal of Vacuum Science \& Technology B 2014, 32, 06F601.

(21) Clark, J. M.; Coleman, R. V. Inelastic electron tunneling spectroscopy of nucleic acid derivatives. PNAS 1976, 73, 1598-1602.

(22) Jaklevic, R. C.; Gaerttner, M. R. Inelastic electron tunneling spectroscopy. experiments on external doping of tunnel junctions by an infusion technique. Applications of Surface Science 1978, 1, 479-502.

(23) Gregory, S. Inelastic tunneling spectroscopy and single-electron tunneling in an adjustable microscopic tunnel junction. Phys. Rev. Lett. 1990, 64, 689-692.

(24) Hipps, K. W.; Mazur, U. Inelastic electron tunneling in aluminum-alumina-gold junctions exposed to thiocyanate ion: sensitive thiocyanate detection. J. Phys. Chem. 1992, 96, 1160-1164.

(25) Higo, M.; Kamata, S. Inelastic Electron Tunneling Spectroscopic Study of Biological Compounds in Human Sweat Adsorbed on Alumina. Anal. Chem. 1994, 66, 818-823.

(26) Kushmerick, J. G.; Lazorcik, J.; Patterson, C. H.; Shashidhar, R.; Seferos, D. S.; Bazan, G. C. Vibronic Contributions to Charge Transport Across Molecular Junctions. Nano Lett. 2004, 4, 639-642. 
(27) Wang, W.; Scott, A.; Gergel-Hackett, N.; Hacker, C. A.; Janes, D. B.; Richter, C. A. Probing Molecules in Integrated SiliconMoleculeMetal Junctions by Inelastic Tunneling Spectroscopy. Nano Lett. 2008, 8, 478-484.

(28) Stipe, B. C.; Rezaei, M. A.; Ho, W. Single-Molecule Vibrational Spectroscopy and Microscopy. Science 1998, 280, 1732-1735.

(29) Franke, K. J.; Pascual, J. I. Effects of electron-vibration coupling in transport through single molecules. J. Phys.: Condens. Matter 2012, 24, 394002.

(30) Dong, J.; Lund, J.; Mehta, R.; Parviz, B. A.; Rahimi, M.; Ryan, D. Using Electron Tunneling for Direct Sequencing of DNA. TechConnect Briefs 2007, 2, 780-783.

(31) Tanaka, H.; Kawai, T. Partial sequencing of a single DNA molecule with a scanning tunnelling microscope. Nature Nanotechnology 2009, 4, 518-522.

(32) Yu, L. H.; Keane, Z. K.; Ciszek, J. W.; Cheng, L.; Stewart, M. P.; Tour, J. M.; Natelson, D. Inelastic Electron Tunneling via Molecular Vibrations in Single-Molecule Transistors. Phys. Rev. Lett. 2004, 93, 266802.

(33) Gupta, R.; Appelbaum, I.; Willis, B. G. Reversible Molecular Adsorption and Detection Using Inelastic Electron Tunneling Spectroscopy in Monolithic Nanoscopic Tunnel Junctions. J. Phys. Chem. C 2009, 113, 3874-3880.

(34) Yu, Q.; Cui, L.; Lequeux, N.; Zimmers, A.; Ulysse, C.; Rebuttini, V.; Pinna, N.; Aubin, H. In-Vacuum Projection of Nanoparticles for On-Chip Tunneling Spectroscopy. ACS Nano 2013, 7, 1487-1494.

(35) van der Wiel, W. G.; De Franceschi, S.; Elzerman, J. M.; Fujisawa, T.; Tarucha, S.; Kouwenhoven, L. P. Electron transport through double quantum dots. Rev. Mod. Phys. 2002, 75, 1-22. 
(36) Gilmore, R. H.; Lee, E. M. Y.; Weidman, M. C.; Willard, A. P.; Tisdale, W. A. Charge Carrier Hopping Dynamics in Homogeneously Broadened PbS Quantum Dot Solids. Nano Lett. 2017, 17, 893-901.

(37) Gilmore, R. H.; Winslow, S. W.; Lee, E. M. Y.; Ashner, M. N.; Yager, K. G.; Willard, A. P.; Tisdale, W. A. Inverse Temperature Dependence of Charge Carrier Hopping in Quantum Dot Solids. ACS Nano 2018, 12, 7741-7749.

(38) Azzaro, M. A.; Dodin, A.; Zhang, D.; Willard, A. P.; Roberts, S. T. ExcitonDelocalizing Ligands Can Speed Energy Migration in Nanocrystal Solids. 2018,

(39) Harrison, P. Quantum Wells, Wires and Dots: Theoretical and Computational Physics of Semiconductor Nanostructures, 3rd ed.; Wiley: West Sussex, England ; Hoboken, NJ, 2010.

(40) Adachi, S. GaAs and Related Materials; WORLD SCIENTIFIC, 1994; pp 546-615.

(41) Landolt, H.; Bornstein, R. Numerical Data and Functional Reationship. Science and Tech. 1987, 22, 118.

(42) Reimann, S. M.; Manninen, M. Electronic structure of quantum dots. Rev. Mod. Phys. 2002, 74, 1283-1342.

(43) MacLeod, M. J.; Goodman, A. J.; Ye, H.-Z.; Nguyen, H. V.-T.; Voorhis, T. V.; Johnson, J. A. Robust gold nanorods stabilized by bidentate N-heterocyclic-carbene-thiolate ligands. Nature Chemistry 2019, 11, 57.

(44) Bender, J. A.; Raulerson, E. K.; Li, X.; Goldzak, T.; Xia, P.; Voorhis, T. V.; Tang, M. L.; Roberts, S. T. Surface States Mediate Triplet Energy Transfer in Nanocrystal-Acene Composite Systems. Journal of the American Chemical Society 2018,

(45) Geva, N.; Shepherd, J. J.; Nienhaus, L.; Bawendi, M. G.; Voorhis, T. V. Morphology of 
Passivating Organic Ligands around a Nanocrystal. The Journal of Physical Chemistry C 2018,

(46) Hastings, W. K. Monte Carlo sampling methods using Markov chains and their applications. Biometrika 1970, 57, 97-109, MSC2010: 65C05 = Monte Carlo methods.

(47) Cho, A. Y.; Arthur, J. R. Molecular beam epitaxy. Progress in Solid State Chemistry 1975, 10, 157-191.

(48) Mata, M. d. 1.; Zhou, X.; Furtmayr, F.; Teubert, J.; Gradečak, S.; Eickhoff, M.; Morral, A. F. i.; Arbiol, J. A review of MBE grown 0D, 1D and 2D quantum structures in a nanowire. J. Mater. Chem. C 2013, 1, 4300-4312.

(49) Jin, R. Quantum sized, thiolate-protected gold nanoclusters. Nanoscale 2010, 2, 343362.

(50) Lee, J.; Yang, J.; Kwon, S. G.; Hyeon, T. Nonclassical nucleation and growth of inorganic nanoparticles. Nature Reviews Materials 2016, 1, 16034.

(51) Harrell, S. M.; McBride, J. R.; Rosenthal, S. J. Synthesis of Ultrasmall and Magic-Sized CdSe Nanocrystals. Chem. Mater. 2013, 25, 1199-1210.

(52) Pu, Y.; Cai, F.; Wang, D.; Wang, J.-X.; Chen, J.-F. Colloidal Synthesis of Semiconductor Quantum Dots toward Large-Scale Production: A Review. Ind. Eng. Chem. Res. 2018, 57, 1790-1802.

(53) Jing, L.; Kershaw, S. V.; Li, Y.; Huang, X.; Li, Y.; Rogach, A. L.; Gao, M. Aqueous Based Semiconductor Nanocrystals. Chem. Rev. 2016, 116, 10623-10730. 